\title{
Why May Repeatability of Highly Flexible Traits Say Little about their Evolutionary Potential?
}

\author{
Daniel E. Naya ${ }^{*}, 1,2$ \\ ${ }^{I}$ Sección Evolución, Facultad de Ciencias \& Centro Universitario de la Regional Este, Universidad de la República, \\ Uruguay \\ ${ }^{2}$ Center for Advanced Studies in Ecology \& Biodiversity, Pontificia Universidad Católica de Chile, Chile
}

\begin{abstract}
A huge advance in our understanding on the relevance of phenotypic plasticity in evolutionary processes has taken place during the last decades. However, there are several specific topics in which the phenotypic plasticity framework has not been deeply considered. One of these topics is our current interpretation of trait repeatability, which is defined as the consistency of a trait over time (typically measured by the intraclass correlation coefficient). Common sense, as well as consideration based on quantitative genetics, suggests that the demonstration of significant repeatability of a trait necessarily precedes any attempt to demonstrate its selective significance. However, this statement is based on the vision that trait variation over time is in some point in between no change (i.e., fixed traits) and completely random variation. Here, I show, through a hypothetical (but realistic) example, that flexible traits able to respond to environmental factors randomly distributed among individuals can evolve through natural selection without showing a significant repeatability value.
\end{abstract}

Keywords: Heritability, natural selection, phenotypic plasticity, repeatability, trait flexibility.

\section{INTRODUCTION}

Phenotypic plasticity refers to changes in organisms' traits due to changes in internal or external environmental conditions (Pigliucci 2001). When these phenotypic changes are reversible over time, the name of phenotypic flexibility is commonly used (Piersma and Drent 2003). During the last three decades, a huge advance in our understanding on the relevance of phenotypic plasticity in evolutionary processes has taken place (Pigliucci 2001, 2005). Nevertheless, we are far from a complete inclusion of this kind of phenomenon into evolutionary theory, and there are several topics in which the phenotypic plasticity framework has not been deeply considered. Here, I will argue that current interpretation of trait repeatability is one of these topics.

Repeatability is defined as the consistency of a trait over time. Operationally, it is usually measured by the intraclass correlation coefficient- i.e., the between-individual component of variance divided by the between-individual component of variance plus within-individual variation- obtained in a one-way ANOVA for several individuals from which two or more measurements are taken (Hayes and Jenkins 1997). Several authors including myself (Lardies et al. 2007), have argued that a trait could evolve by natural selection if, and only if, it is repeatable (Bech et al. 1999; Nespolo et al. 2003, Chown et al. 2006; Nespolo and Franco 2007, Cortés et al. 2009). The rationale behind this idea is

*Address correspondence to this author at the Sección Evolución, Facultad de Ciencias, Universidad de la República, Iguá 4225, Montevideo 11400, Uruguay; Tel: (0598) 2 5258618; Fax: (0598) 2 5258617; E-mail: dnaya@fcien.edu.uy

Handling Editor: Helena Freitas fairly simple: a trait that changes randomly over time, if it can be considered a trait after all, cannot be consistently selected in the long term. In addition, quantitative geneticists have demonstrated that repeatability sets the upper limit of heritability, since repeatability includes all genetic components and permanent environmental variances in the numerator, while heritability only includes additive genetic differences between organisms (Falconer and Mackay 1997, Lynch and Walsh 1998). This reinforces the idea that the demonstration of significant repeatability of a trait necessarily precedes any attempt to demonstrate its selective significance (Nespolo and Franco 2007). However, all these statements are based on the vision that trait variation over time is in some point in between no change (i.e., fixed traits; Fig. 1A) and completely random variation (Fig. 1B). I will show, through a simple hypothetical example that flexible traits which are able to respond to enviromental factors randomly distributed among individuals can evolve through natural selection without showing a significant repeatability value (Fig. 1C).

\section{THE HYPOTHETICAL EXAMPLE}

As a target trait I chose the metabolic rate (MR), because this variable is considered one of the most important physiological traits in animals, being related with several aspects of energy budget. At large scales, body mass and taxonomic affiliation are the major determinants of MR, while at smaller scales (e.g., intra-specific) several additional factors can affect this variable (Muller and Diamond 2001). One of these factors is the quality of the food being consumed (Veloso and Bozinovic 1993). Thus, in this example, MR will be the target trait and diet quality the environmental factor that changes over time. For the sake of 


\section{Current vision based in fixed trait}

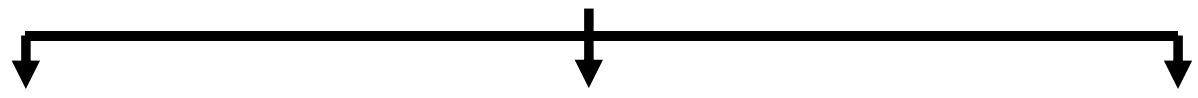

(A) High repeatibility
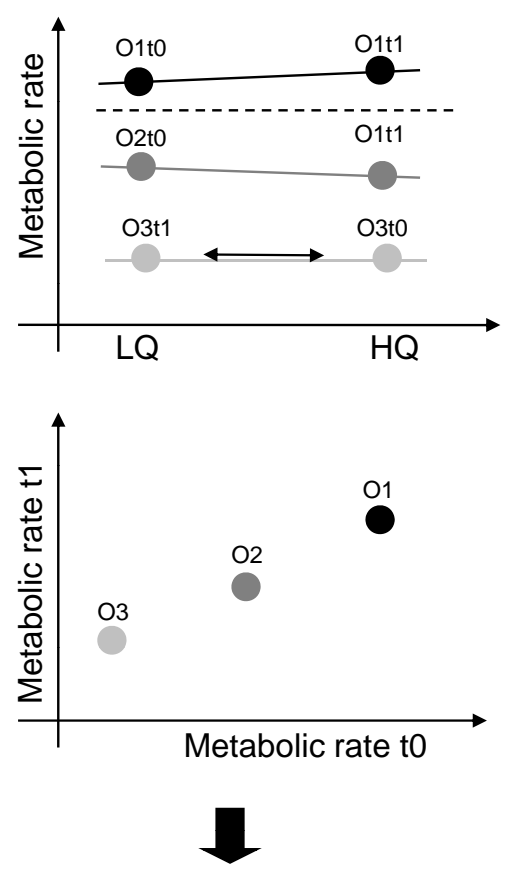

Evolution is possible (correct)
(B) Low repeatibility (randomly changes)
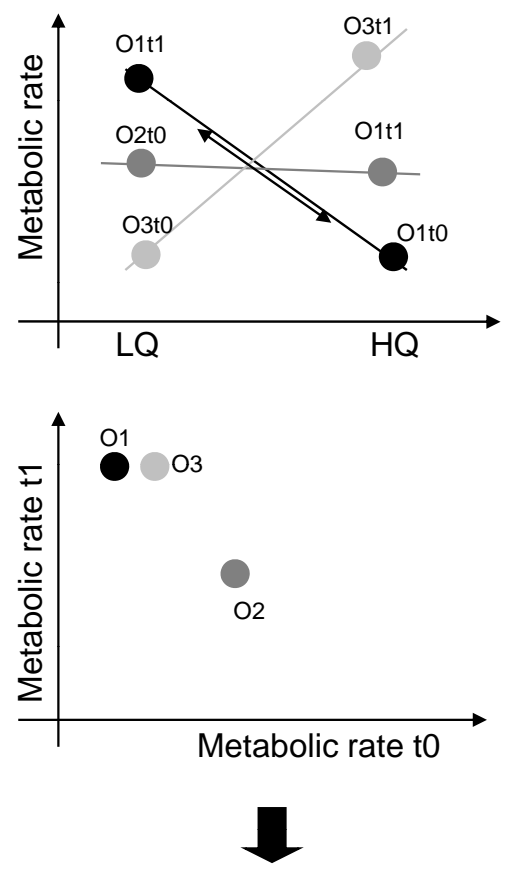

Evolution is not possible (correct)
(C) Low repeatibility (adaptive plasticity)
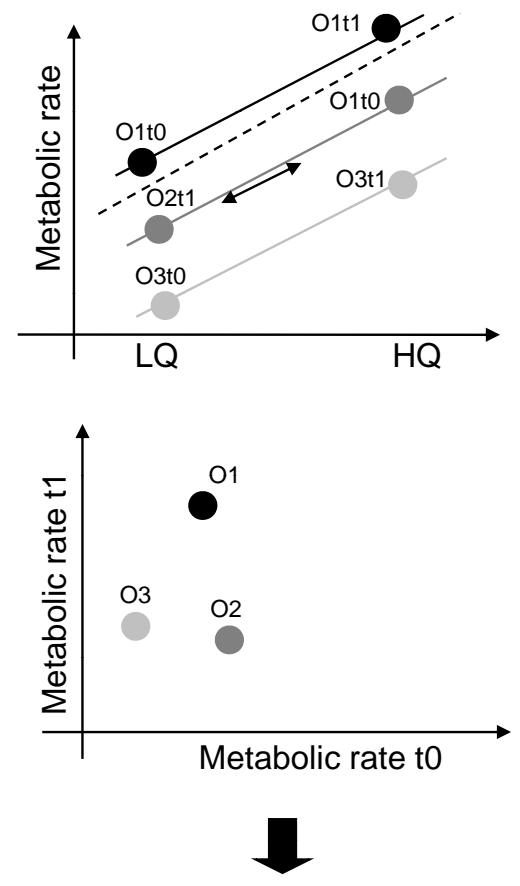

Evolution is not possible (false)

Fig. (1). Three hypothetical situations showing the relationship between reaction norms, trait repeatability and trait potential to evolve by natural selection. Reactions norms are showed in the upper panels, while correlation between trait values at different times (repeteability) are showed in the lower panels. Abbreviations: $\mathrm{LQ}=$ low quality diet, $\mathrm{HQ}=$ high quality diet, $\mathrm{Oi}=$ organism $\mathrm{i}$, tj $=$ time $\mathrm{j}$. Dotted lines represent hypothetical selection events and double-head arrows indicate those organisms that were first measured in the HQ environment (in contrast to the other two individuals).

simplicity, only three organisms (O1 to O3), whose metabolic rates were assessed at two different times ( $\mathrm{t} 0$ and $\mathrm{t} 1$ ), will be considered.

Now, suppose that the physiological behavior with higher performance in the population is to modulate MR according to the (mean) diet quality consumed during a certain time (say last weeks), increasing MR values when food quality is higher. Thus, given some temporal variability in the quality of resources, the population will be dominated by flexible organisms that show positive reaction norms (Fig. 1C). In other words, those organisms that do not change their MR (Fig. 1A), or change it randomly (Fig. 1B), will disappear from the population. The second assumption of this example is that resources of different quality are randomly distributed in time among individuals (i.e., all organisms have the same probability of obtaining low or high quality resources at a given time). It means that "well adapted" flexible individuals could show higher or lower MR according to their "good or bad luck" in the resource sorting.

Under the above-described scenario, an experimenter who tries to estimate the repeatability of MR assessing this variable at different times will fail to observe a significant value. It will be so, simply because the expected value of MR for a given organism at any time is determined by the quality of the diet consumed, which follows a random distribution among organisms (Fig. 1C). Probably, the experimenter will erroneously conclude that MR evolution by natural selection is not possible.

A similar, but real, case was recently published for the marsupial Monito del Monte, Dromiciops gliroides, by Cortés et al. (2009). When these authors analyzed repeatability for some energetic variables, they found very low nonsignificant values, concluding that these traits "do not exhibit evolutionary potential". It is noteworthy their conclusion for the case of thermal conductance. After quoting different lines of evidence suggesting that flexiblity in this trait is adaptative (e.g., seasonal adjustments, changes during acclimatation periods), they stated: "This phenotypic plasticity suggests an adaptive value for thermal conductance. However, this contrasts with the almost absolute absence of inter-individual variation in this trait in $D$. gliroides as only negative repeatability estimations were found (i.e., the actual value of repeatability was near zero). In other words, thermal conductance in D. gliroides would not respond to selection". 


\section{FINAL REMARKS}

Repeatability represents the proportion of the overall variance observed in a trait due to variation between individuals. Under the classical vision, a low repeatability value indicates that the trait changes randomly within individuals over time and, consequently, that it cannot be the target of natural selection. However, considering phenotypic flexibility, intraindividual variation can represent a substantial part of the overall variance and, at the same time, the trait can be the target of selection. Note that this does not imply that relevant plastic traits should not be repeatable over time. By contrast, evidence for high repeatability values in energetic traits was recently demonstrated (Nespolo and Franco 2007).

In a very interesting report, Dohm (2002) used path models to describe five situations in which repeatability is not expected to set the upper limits to heritability: (1) repeated measures reflect different traits genetically, (2) common environmental effects oppose genetic effects, (3) correlation between temporary environments, (4) interactions between genotype and environment, and (5) influence of maternal effect on measured traits. Although the author states that phenotypic plasticity could be modelled as a particular case of the genotype-environment interaction, all the cited examples refer to the last kind of phenomena. From this perspective, this note is just an attempt to provide a hypothetical (but realistic) example showing how a flexible trait, for which there is no genotype-environment interaction, could also violate the assumptions needed to interpret repeatability as the upper limit to heritability.

\section{ACKNOWLEDGEMENTS}

Many thanks to all the students and teachers of the "I Latin American School of Evolution" for their stimulus, to Carolina Abud for grammar corrections, to Antonio B. Ávila for the use of his facilities, and to Hugo Naya and two anonymous referees for valuable comments on the manuscript. This work was funded by Comision Sectorial de
Investigación Cientifica (CSIC, Uruguay), Programa de Desarrollo de las Ciencias Basicas (PEDECIBA, Uruguay) and FONDAP 1501-0001 Program 1 (Conicyt, Chile).

\section{REFERENCES}

Bech, C, Langseth, I \& Gabrielsen, GW (1999) Repeatability of basal metabolism in breeding female kittiwakes Rissa tridactyla. Proceeding of the Royal Society of London B, 266, 2161-67.

Chown, SL, Gibbs, AG, Hetz, SK, Klok, CJ, Lighton, JRB \& Marais, E (2006) Discontinuous gas exchange in insects: a clarification of hypotheses and approaches. Physiological and Biochemical Zoology, 79, 333-43.

Cortés, P, Quijano, SA \& Nespolo, RF (2009) Bioenergetics and interindividual variation in physiological capacities in a relict mammal - the Monito del Monte (Dromiciops gliroides). The Journal of Experimental Biology, 212, 297-304.

Dohm, MR (2002) Repeatability estimates do not always set an upper limit to heritability. Functional Ecology, 16, 273-80.

Falconer, DS \& Mackay, TFC (1997) Introduction to Quantitative Genetics, 4th ed, Longman Scientific and Technical: Edinburgh.

Hayes, JP \& Jenkins, SH (1997) Individual variation in mammals. Journal of Mammalogy, 78, 274-93.

Lardies, MA, Naya, DE, Berrios, P \& Bozinovic, F (2008) The cost of living slowly: metabolism, Q10 and repeatability in a South American harvestman. Physiological Entomology, 33, 193-99.

Lynch, M \& Walsh, B (1998) Genetics and Analysis of Quantitative Traits, 1st ed, Sinauer Associates: Massachusetts.

Mueller, P \& Diamond, J (2001) Metabolic rate and environmental productivity: well-provisioned animals evolved to run and idle fast. Proceeding of the National Academy of Science USA, 98, 1255054.

Nespolo, RF, Lardies, MA \& Bozinovic, F (2003) Intrapopulationvariation in the standard metabolic rate of insects: repeatabilitym thermal dependence and sensitivity (Q10) of oxygen consumption in a cricket. The Journal of Experimental Biology, 206, 4309-15.

Nespolo, RF \& Franco, M (2007) Whole-animal metabolic rate is a repeatable trait: a meta-analysis. The Journal of Experimental Biology, 210, 2000-5.

Piersma, T \& Drent, J (2003) Phenotypic flexibility and the evolution of organismal design. Trends in Ecology and Evolution, 18, 228-33.

Pigliucci, M (2001) Phenotypic Plasticity: Beyond Nature and Nurture, 1st ed, John Hopkins University Press: Baltimore.

Pigliucci, M (2005) Evolution of phenotypic plasticity: where are we going now? Trends in Ecology and Evolution, 20, 481-86.

Veloso, C \& Bozinovic, F (1993) Dietary and digestive constraints on basal energy metabolism in a small herbivorous rodent. Ecology, 74, 2003-10.

Received: October 27, 2009

Revised: February 23, 2010

Accepted: February 24, 2010

(C) Daniel E. Naya; Licensee Bentham Open.

This is an open access article licensed under the terms of the Creative Commons Attribution Non-Commercial License (http://creativecommons.org/licenses/bync/3.0/), which permits unrestricted, non-commercial use, distribution \& reproduction in any medium, provided the work is properly cited. 